\title{
Effects of Linear Holding for Reducing Additional Flight Delays without Extra Fuel Consumption
}

\author{
Yan Xu and Xavier Prats \\ Technical University of Catalonia, Castelldefels 08860, Barcelona (Spain)
}

\begin{abstract}
This paper presents an approach to implement linear holding (LH) for flights initially subject to ground holding, in the context of Trajectory Based Operations. The aim is to neutralize additional delays raised from the lack of coordination between various traffic management initiatives (TMIs) and without incurring extra fuel consumption. Firstly, motivated from previous works on the features of LH to absorb delays airborne, a potential applicability of LH to compensate part of the fixed ground holding is proposed. Then, the dynamic adjustment of LH in response to TMIs-associated tactical delays is formulated as a multi-stage aircraft trajectory optimization problem, addressing both pre- and post-departure additional delays. Results suggest that additional delays of $25 \mathrm{mins}$ in a typical case study can be totally recovered at no extra fuel cost. A notable extent of delay reduction observed from the computational experiments further supports the benefits of LH for reducing different combinations of additional delays without consuming extra fuel.
\end{abstract}

Keywords: air transportation, linear holding, speed reduction, trajectory optimization, air traffic flow management

\section{Introduction}

In the recent 16th ATIO (AIAA) conference, Bilimoria [1] presented an analysis of the additional delays experienced by flights subject to ground holding for Ground Delay Programs (GDPs) or Airspace Flow Programs (AFPs). Statistic results obtained from five airports of arrivals suffering the most pre-departure ground holding in 2015 were shown, suggesting that the additional delays of those EDCT (Expect Departure Clearance Time) affected flights were substantially larger in four of the five airports (about 
two to three times on average) than for arrivals that were not subject to ground holding. At the same conference, a similar analysis of "double delay" (or "double penalty"), due to the interaction between GDPs and arrival metering (terminal scheduling delays), was presented by Evans and Lee [2], providing a deep dive into the underlying causes of those double delays and the circumstances in which they occur in real operations.

Imagine a flight held on ground due to a GDP/AFP, before being rerouted around a thunderstorm, and then subject to the Miles-in-Trail (MIT) as it passes through a congested sector, as described in [3]. The joint impact of all these initiatives together, however, may not be well coordinated and eventual inequities in their implementation may be perceived for the airspace user. Under current operations, delays assigned by the GDP/AFP are normally transferred from the area of affected capacity to the departure airport, while imposed entirely on the EDCT, prior to take-off. As a consequence, it is possible that some unnecessary delays may have been performed through the ground holding, before the controlled flight encounters other initiatives yielding delays likewise, which again pushes back its final arrival time.

The above discussions might point to a drawback of ground holding: its low flexibility, especially in terms of integrating among various Traffic Management Initiatives (TMIs), as reported by $[4,5]$, being a real problem in the United States National Airspace System (NAS). Even so, ground holding is still preferred nowadays to absorb delays because less fuel consumption is incurred if compared with typical airborne holding. In order to overcome some of the ground holding drawbacks, Delgado et al. $[6,7,8]$ proposed a cruise speed reduction strategy aimed at partially absorbing delays airborne, where ground delayed flights were allowed to cruise at the lowest possible speed in such a way the specific range remained the same. In this situation, the fuel consumption kept unchanged while some linear holding (LH) was performed in compensation with the reduced ground holding. Concretely, differentiating from typical airborne holding, which would consume more fuel due to the extended flight track (such as vectoring or using holding patterns), this LH is also performed airborne but done progressively by flying slower along the original planned route whilst having no extra fuel consumed than initially scheduled.

As the core method to perform LH, speed reduction is one of the speed control strategies that have proven effective for several Air Traffic Management (ATM) scenarios. For instance, $[9,10]$ presented a speed control approach for transferring delay away from the terminal to the en route phase, 
from which significant fuel saving on a per flight basis was yielded, and the performance of GDP was reported improved through a dynamic speed adjustment mechanism. In [11], a pre-tactical speed control was applied to prevent aircraft from performing holding patterns when arriving at a congested airspace, improving both flight efficiency and controller workload level. More widespread applications for conflict management have been under research for decades [12], where the speed control strategy was used, in addition to other effective manners such as path stretching or flight level adjustment for instance.

More recently, the LH strategy was further extended by the same authors of this paper in [13], where, through using trajectory optimization techniques, the whole flight profile including climb, cruise and descent phases were subject of realizing LH, being a remarkable increase appreciated in terms of the maximum amount of airborne delay that can be generated without extra fuel consumption. Yet, as a primary study in this regard done by the authors, reference [13] mainly focused on the inclusion of climb/descent flight phases into the LH strategy, without paying any attention on the discussions of the subsequent applicability (e.g., a delay recovery process) and the effects on potential fuel savings. This paper, as a result, is devoted to take advantage of the former research and complement the side of potential applications.

With the paradigm shift proposed by NextGen and SESAR programs, evolving from an airspace-based ATM to Trajectory Based Operations (TBO), the proposed LH could be expected to provide a high flexibility with regard to the cost-based delay management. The purpose of this paper is to implement LH to substitute part of the ground holding, adjusted dynamically in response to potential TMIs that might produce tactical delays during preand post-departure phases. In such a way, our aim is to reduce the additional delays as much as possible at no extra fuel cost. For this purpose, an optimal trajectory generation technique is used to formulate each of the steps of the implementation, followed by a case study illustrating in detail the effects of dynamic adjustment of LH to a specific flight, as well as computational experiments on the capability of delay recovery with respect to different TMIs-associated delay combinations.

\section{Motivation}

Fig. 1 illustrates some key flight-related events (blue rectangles) and respective time intervals. In [1], historical flight operations from five airports 
(LGA, SFO, EWR, JFK, PHL), whose arrivals experienced the most predeparture ground holding in 2015, were examined computing the variance between scheduled times and actual times. According to this study, each of the flight-related events of Fig. 1 can be associated with a possible additional delay event (red rectangles in the figure).

Consider a particular flight affected by a GDP (or an AFP, where the affected area is not at the destination airport but somewhere en-route). To be more precise, a GDP is usually implemented at airports where capacity has been reduced because of weather (such as low ceilings, thunderstorms or wind) or when demand exceeds capacity for a sustained period. The FAA (Federal Aviation Administration) assigns arrival "slots" to aircraft based on the available capacity and flight arrival times, and adds delay in sequential order until demand equals capacity. This process affects all flights within the defined "scope" (i.e., which flights are captured) of the program, which in turn can be specified by distance, by tier, or by time. For more details about the procedure, the readers may refer to [14]. Nevertheless, it should be noted that the application of the proposed strategy should not be limited for GDP only. We choose the GDP as an illustrative example, as it is nowadays widely used in real practice.

As mentioned above, because of the capacity reduction at the destination airport, the arrival time becomes "controlled" and postponed by a certain GDP delay (from "Scheduled Flight" to "Scheduled Flight in GDP" in Fig. 1). At present, time-of-arrival control is not enforced and a time-of-departure control is preferred. The reason is because a departure time is actually enforceable, being much more difficult to enforce the arrival time with current navigation and guidance technology. Thus, the assigned delay is entirely transferred from the arrival airport to the departure in the form of ground holding (GH) also to avoid (relatively) costly airborne holding, and to obtain a parallel shift on the scheduled arrival time (Wheels On in the figure).

Due to the likely additional (and unforeseen) delays, however, the already delayed time of Wheels On could be delayed again, as from "Scheduled Flight in GDP" to "Actual Flight in GDP". In such a situation, extra fuel has to be consumed by increasing flight speed if these unforeseen delays are to be recovered (as usually done nowadays by some airlines). Furthermore, it is also a common practice for some airlines to speed-up after departure to even recover part of the initially assigned GDP delay, since arrival times are not (yet) enforced.

Worth noting that some types of additional delays, such as those asso- 


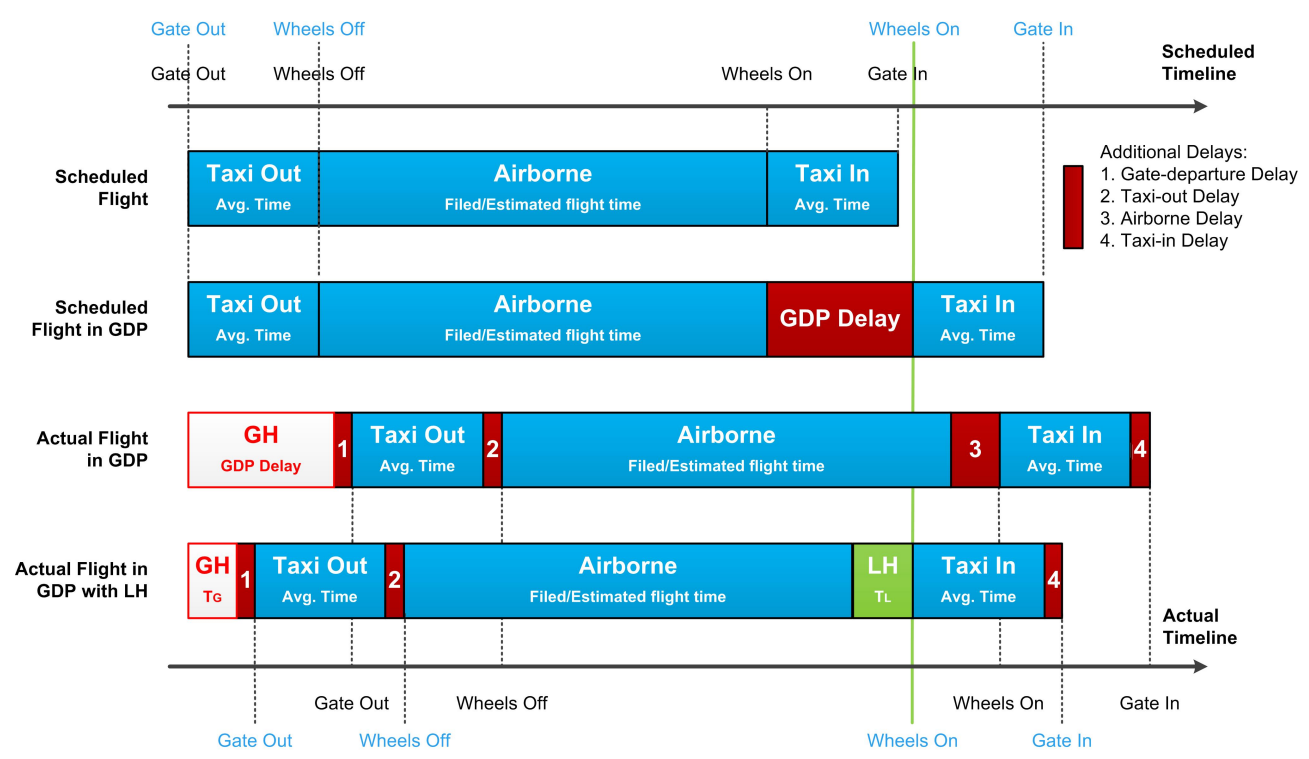

Figure 1: Schematic diagram of additional delays subject to a GDP with the LH applicability.

ciated with gate-departure and taxi-out/in, are being targeted heavily by research and development efforts in (airport) surface management systems (see $[15,16]$ for instance and the references therein). If one day these delays are well reduced as a result, then part of the motivation for this paper might disappear. Nevertheless, despite of all the efforts devoted to reduce delays, there will always be uncertainties in the trajectories and this LH strategy shall help absorb unplanned delays and thus enhance the predictability.

In this paper we propose to shorten the ground holding and absorb part of the assigned delay airborne by means of linear holding ( $\mathrm{LH})$, i.e. by flying slower than initially planned. This is depicted in Fig. 1 as "Actual Flight in GDP with LH" and at the same time the scheduled arrival time in GDP is met (green line). Thanks to the flexibility of this method, every time an additional delay is encountered during the execution of the flight, the required LH can be updated through speed control. In this way the extra (unforeseen) delay can be neutralized adding only, in theory, taxi-in time uncertainties into the final gate-in time.

Wrapping up, Fig. 1 aims to illustrate the effects of incurring additional (and not desired) delay due to stochastic downstream events and how the LH strategy proposed in this paper could be used to partially or totally 
neutralize them. It should be noted, however, that this strategy requires an enforcement of arrival times and therefore only makes sense in the near future scenario, where we could expect accurate 4D navigation and guidance equipment (in line with the TBO concept).

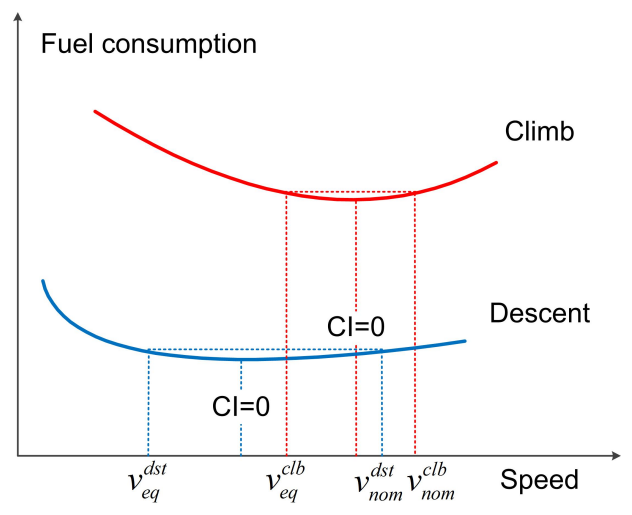

(a) Climb and Descent

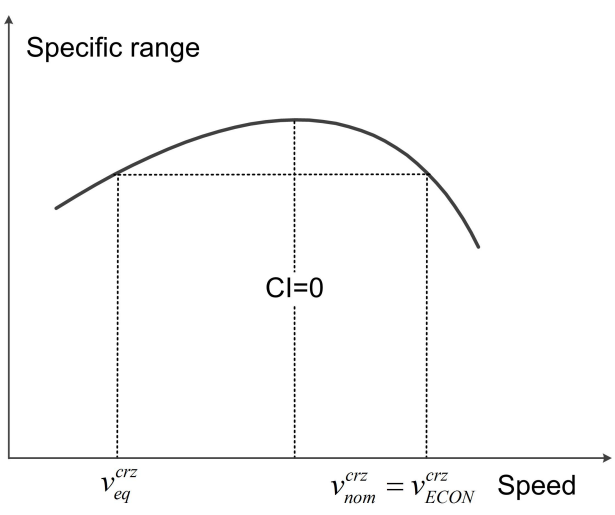

(b) Cruise

Figure 2: Definition of equivalent speeds $\left(V_{e q}\right)$ in climb, descent and cruise phases, and their corresponding nominal speeds $\left(V_{\text {nom }}\right)$.

The speed reduction needed to perform LH is based on the equivalent speed concept, which was introduced in [13] and shown in Fig. 2. Notionally, this equivalent speed is defined as the speed that produces the same amount of fuel consumption than the nominal speed (in climb/descent) or the same amount of specific range (i.e. distance flown per unit of fuel) in cruise. By flying at these speeds, the LH can be realized with no extra fuel consumption if compared with the nominal flight.

In this paper, the initially scheduled flight is regarded as the nominal flight. Since airline operators also consider time-related costs (e.g., guarantee of connecting passengers, flight crew payments) when planning their flights, speeds higher than the minimum fuel speed are usually preferred despite the associated extra fuel costs. This trade-off between time and fuel is typically expressed by the Cost Index (CI), an input parameter featuring all current on-board Flight Management System (FMS) [17]. The higher the CI is, the more importance will be given to flight time and the faster the scheduled flight speed will be.

The speed margins between the nominal and equivalent speed not only depend on the chosen CI (affecting directly the value for the nominal speed), 
but also depend on the curves of fuel consumption (or specific range), as shown in Fig. 2, which in turn should rely on aircraft mass, aircraft performance parameters and atmospheric magnitudes. Consequently, a practical way to obtain these notional equivalent speeds in order to maximize the amount of LH that can be done at no extra fuel cost is to formulate and solve a continuous optimal control problem, as explained in the next section.

\section{Optimal trajectory generation}

The optimization of aircraft 4D trajectories requires the definition of a mathematical model representing aircraft dynamics and performance, along with a model for certain atmospheric variables. In this paper, a point-mass dynamic model, an enhanced performance model using manufacturer performance data and the International Standard Atmosphere (ISA) have been considered. For more details on this implementation, the readers may refer to $[18]$.

\subsection{Scheduled trajectory filed into a GDP with an EDCT assigned}

A generic aircraft trajectory can be divided into several segments $i \in$ $[1, \ldots, N]$. For each segment defined over the time window $\left[t_{0}^{(i)}, t_{f}^{(i)}\right]$ the state vector $\boldsymbol{x}^{(i)}=\left[\begin{array}{lll}v & s & h\end{array}\right]^{T}$ is composed by the true airspeed (TAS), along path distance, altitude and mass of the aircraft, respectively; the control vector $\boldsymbol{u}^{(i)}=\left[\begin{array}{ll}T & \gamma\end{array}\right]$ includes the aircraft thrust and flight path angle [18]; and a parameter $\boldsymbol{p}^{(i)}$ vector of variables that are not time depended is also defined.

For the initially scheduled flight, the objective of trajectory optimization is to minimize a compound cost function $J$ over the whole time window $\left[t_{0}^{(1)}, t_{f}^{(N)}\right]$ as follows:

$$
J=\int_{t_{0}^{(1)}}^{t_{f}^{(N)}}(F F(t)+C I) \mathrm{d} t
$$

where $F F(t)$ is the fuel flow and $C I$ the Cost Index, combined as to reflect the direct operating costs.

The optimization constraints come from different aspects, while the first important set are the dynamics of the aircraft itself (point-mass dynamic model). Then, some algebraic event constraints fixing the initial $\boldsymbol{x}\left(t_{0}^{(1)}\right)$ and

final $\boldsymbol{x}\left(t_{f}^{(N)}\right)$ state vector must be satisfied. In this paper, the initial and final points are taken, respectively, at the moment the slats are retracted (after 
taking off) and extended (before landing). The remaining parts of takeoff and approaching are not optimized due to the heavy constraints from operational procedures.

Some bounds (known as box constraints) on the control variables are specified as follows:

$$
\gamma_{\min } \leq \gamma \leq \gamma_{\max }
$$

where $\gamma_{\min }$ and $\gamma_{\max }$ are aircraft dependent scalars. However, the maximum $T_{\max }$ and minimum $T_{\min }$ thrust are not scalars but functions of the state variables. Therefore, this control is bounded by additional path constraints:

$$
T_{\min } \leq T \leq T_{\max }
$$

Similarly, box constraints for the state variables are not required, since they are bounded by generic path constraints on auxiliary variables such as the Mach number $(M)$ and the Calibrated Airspeed (CAS, $\left.V_{C A S}\right)$ :

$$
M_{G D} \leq M \leq M M O ; V_{G D} \leq V_{C A S} \leq V M O
$$

where $M M O$ and $V M O$ are the maximum operational Mach and CAS, respectively, and $M_{G D}$ and $V_{G D}$ are green dot speeds [19], which approximate the best lift to drag ratio speed in clean configuration.

In order to ensure the continuity of the trajectory composed by different segments, link constraints must be defined at the final point and initial point of each segment, on all the state variables:

$$
\boldsymbol{x}^{(i)}\left(t_{f}^{(i)}\right)=\boldsymbol{x}^{(i+1)}\left(t_{0}^{(i+1)}\right) ; i=1, \ldots, N-1
$$

Next, additional path and event constraints on the flight profile, which are flight segment dependent, must be considered in order to guarantee the optimized trajectory be consistent with typical ATM operations and regulations. These constraints are summarized in Fig. 3.

It should be noted that before each cruise flight level, a short cruise segment less than $1 \mathrm{~min}$ is added, allowing in this way proper speed adjustments (as shown with the blue lines in Fig. 3). A similar segment is also added at the end of the last cruise segment. More mathematical details on the formulation of this flight profile can be found in [18].

In addition to the flight vertical (and speed) profile, a flight route must be defined either in terms of Great Circle Distance (GCD) between city-pair 


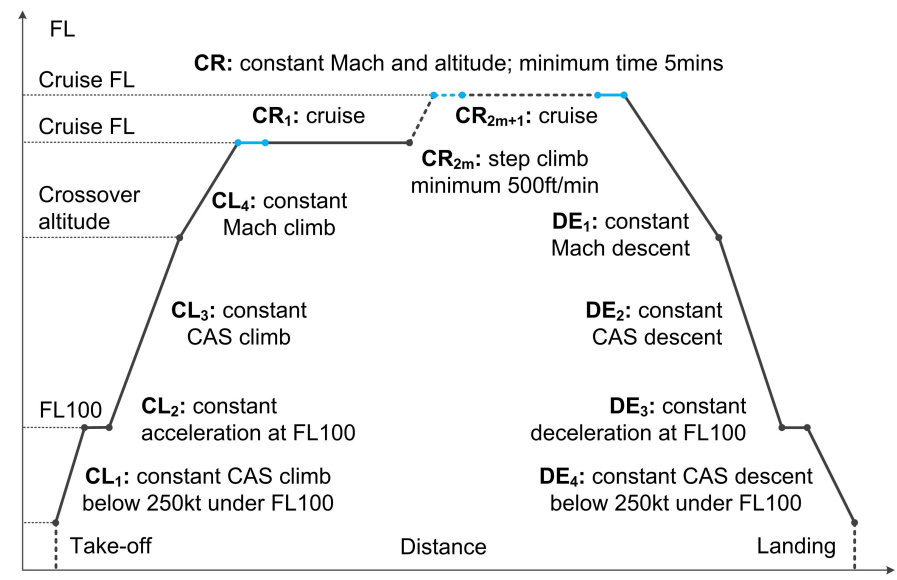

Figure 3: Simulated flight profile, divided in different segments to take into account specific trajectory constraints.

airports, or by using air traffic services (ATS) route waypoints and published procedures (such as standard instrumental departures and arrivals).

To find the optimal solution of the formulated optimal control problem, direct collocation methods [20] are used in this paper, which discretize the time histories of control and state variable at a set of nodal or collocation points, transforming the original continuous (infinite) optimal control problem into a (discrete and finite) nonlinear programming (NLP) optimization problem. The new finite variable NLP problem is solved by using solvers CONOPT (as NLP) and SBB as MINLP (mixed integer nonlinear programming), both bundled into the GAMS software suite [21, 22]. The whole process is briefly presented in Fig. 4.

Finally, as stated in Sec. 2, the difference between nominal flight and the one performing entirely ground holding lies only on the timeline, maintaining the remaining 3D trajectory unchanged. Therefore the initially assigned GDP delay could be added directly on the flight's EDCT.

\subsection{Scheduled trajectory with LH meeting GDP delay at final arrival}

For the flight performing LH the objective function is switched from Eq. 1 to Eq.6, in order to minimize ground holding and to leave enough time to neutralize possible additional delays before departure $\left(t_{0(n o m)}^{(1)}\right.$ is the initial time of nominal flight).

$$
J=t_{0}^{(1)}-t_{0(\text { nom })}^{(1)}
$$




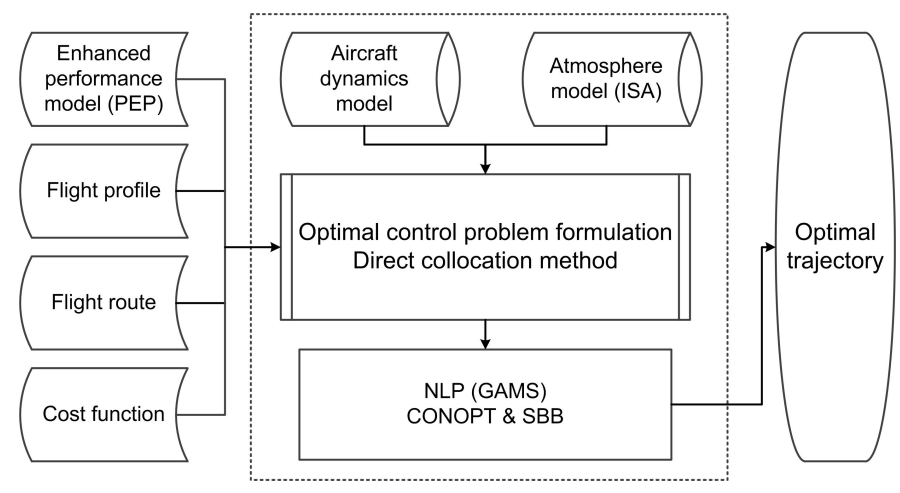

Figure 4: Schematic process in generating the optimal aircraft trajectory.

This new optimization problem is subject to the same constraints in generating nominal trajectory listed in Sec. 3.1, along with the following restrictions:

$$
\begin{gathered}
t_{0}^{(1)}-t_{0(\text { nom })}^{(1)} \geq 0 \\
t_{f}^{(N)}=t_{f(\text { nom })}^{(N)}+\Delta t_{G D P} \\
\int_{t_{0}^{(1)}}^{t_{f}^{(N)}} F F(t) d t \leq m\left(t_{0(\text { nom })}^{(1)}\right)-m\left(t_{f(n o m)}^{(N)}\right)
\end{gathered}
$$

Eq. 7 ensures the departure time not earlier than that initially scheduled. Eq. 8 specifies that the assigned GDP delay $\left(\Delta t_{G D P}\right)$ is fully realized at the arrival. Eq. 9 imposes the maximum fuel consumption allowed, which equals to the amount consumed by the nominal flight, where $m\left(t_{0(\text { nom })}^{(1)}\right)$ and $m\left(t_{f(n o m)}^{(N)}\right)$ are, respectively, the initial and final mass of aircraft (whose difference is the fuel burned on trip).

In this case, the ideal scheduled trajectory performing LH will be generated. If none of the additional delay occurs later, the flight will endure less ground holding but still meet the same arrival slot at the GDP airport exactly as performing an entire ground holding, while consuming no extra fuel. 


\subsection{Actual trajectory after pre-departure additional delays experienced}

After ready for gate-out, assume an additional delay $\Delta t_{1}$ arises in the gate-departure process (see Fig. 1), followed by another one $\Delta t_{2}$ during taxiout phase before departure. It should be noted that these delays can be negative, and an airborne holding may be needed if the scheduled LH has reached its maximum. However, considering the same situation applies in the context of ground holding only, and assessing the impact of these negative delays are out of the scope of this paper and are left for future work.

Then, the initially scheduled trajectory can be updated with a new objective function, minimizing the difference between the arrival time and the final time of the ideal scheduled trajectory with $\mathrm{LH}\left(t_{f(L H)}^{(N)}\right)$ :

$$
J=t_{f}^{(N)}-t_{f(L H)}^{(N)}
$$

The same constraints for the nominal flight and Eq. 9 apply in such situation, along with the following additional restrictions:

$$
\begin{gathered}
t_{f}^{(N)}-t_{f(L H)}^{(N)} \geq 0 \\
t_{0}^{(1)}=t_{0(L H)}^{(1)}+\Delta t_{1}+\Delta t_{2}
\end{gathered}
$$

Eq. 11 ensures the final arrival time not earlier than the assigned slot at GDP airport, which is $t_{f(n o m)}^{(N)}+\Delta t_{G D P}$, as stated in Eq. 8. Eq. 12 updates the departure time with regards to the amount of additional delays experienced on ground.

\subsection{Actual trajectory after post-departure additional delays experienced}

When airborne, additional delays $\Delta t_{3}$ may arise (see Fig. 1); due to TMIs, such as speed instructions to meet MIT restrictions; or air traffic control (ATC) maneuvers, such as path stretching (radar vectoring) for separation purposes, or air holding patterns (usually in a shape of racetrack pattern, involving two turns and two legs, used to keep an aircraft within a prescribed airspace with respect to a geographic fix [23]) to tactically absorb large delays. The effects from each of them to aircraft trajectory may vary substantially, and because flights are typically under real-time control from these TMIs, there is rarely any space for trajectory optimization.

Accordingly, the short flight segment during this phase is regarded as a black box in this paper, being only time and mass (fuel) discretized by fixed values, while keeping other variables continuous and unchanged. 
In this case, the objective function is still as presented in Eq. 10, but the initial point of the optimization problem is moved to the phase where this airborne delay occurs (i.e., initial time is defined as $t_{A D}^{(1)}$ ). In addition, the flown trajectory must also be fixed, along with added constraints below:

$$
\begin{gathered}
t_{A D}^{(1)}=t_{A D(\text { LHpre })}^{(1)}+\Delta t_{3} \\
m\left(t_{A D}^{(1)}\right)-m\left(t_{A D(\text { LHpre })}^{(1)}\right)=-F F\left(t_{A D(\text { LHpre })}^{(1)}\right) \Delta t_{3} \\
\int_{t_{A D}^{(1)}}^{t_{f}^{(N)}} F F(t) d t \leq m\left(t_{A D(\text { LHpre })}^{(1)}\right)-m\left(t_{f(\text { nom })}^{(N)}\right)
\end{gathered}
$$

where $t_{A D(\text { LHpre })}^{(1)}$ denotes the time when the (unforeseen) airborne delay starts. Eq. 13 updates the initial time of optimization with the airborne delay added. Eq. 14 deducts the fuel consumed by using current fuel flow multiplied by the delayed time, while Eq. 15 specifies that this part of fuel caused from airborne delay is not taken account into the premise that no extra fuel is allowed.

\section{Numerical results}

An illustrative example is shown in this section, with results obtained for a specific case study, where a scheduled flight, from ATL (Atlanta) to LGA (LaGuardia) airports with a great circle distance of $662 \mathrm{~nm}$, is captured in a GDP list issued from LGA and assigned with a delay of 40mins. An Airbus A320, a common two-engine, narrow-body transport aircraft, is assumed to execute this flight mission. For the nominal trajectory (scheduled flight) generation, a typical passenger load factor of $81 \%$ has been considered [6], along with a CI of $30 \mathrm{~kg} / \mathrm{min}$ in the FMS.

Some assumptions have been taken: 1) each type of additional delay, is set as a fixed number according to the average statistic value found in [1], i.e., gate-departure: 9mins, taxi-out: 9mins, airborne: 7mins; 2) airborne delay occurs at the middle of the flight distance, and ends at the same place (i.e., at place of $331 \mathrm{~nm}$ after departure); 3) no wind conditions are considered; 4) only even flight levels are used (FL260 as the lowest altitude); and 5) cruise step climbs are allowed (if any) with $2000 \mathrm{ft}$ steps.

In accordance with the flight process, as discussed in Sec. 3, the computational experiment has been conducted in five main steps: 
- Nominal (nom): Initially scheduled flight minimizing direct operating costs (equation 1);

- Step1: Scheduled trajectory filed into a GDP with an EDCT assigned;

- Step2: Scheduled trajectory with LH meeting GDP delay at final arrival;

- Step3: Actual trajectory after pre-departure additional delays experienced; and

- Step4: Actual trajectory after post-departure additional delays experienced.

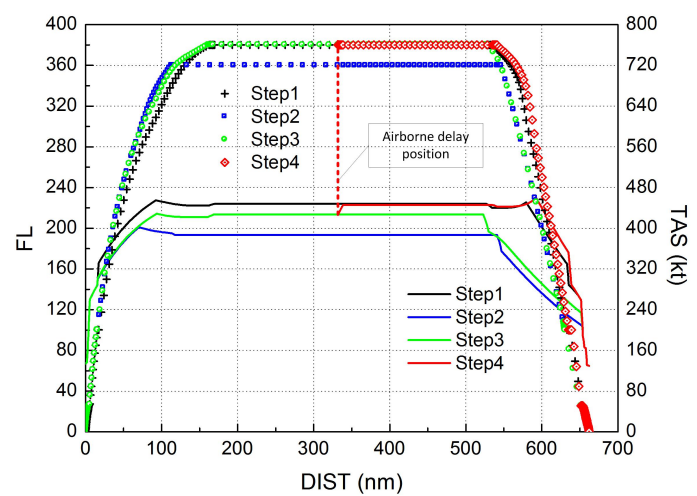

(a) Altitude and speed profiles

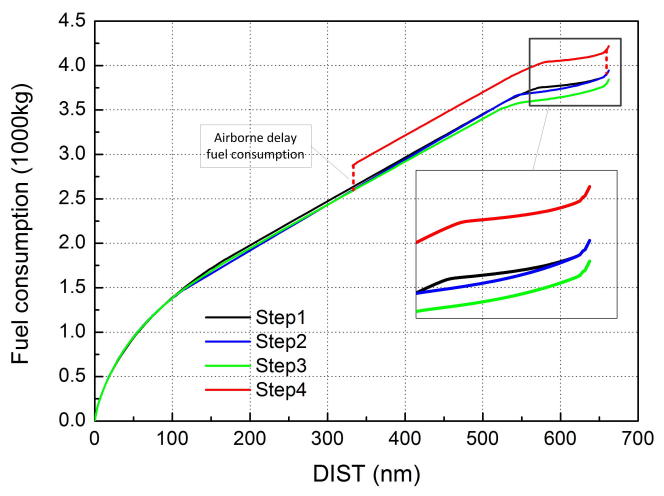

(c) Fuel consumption

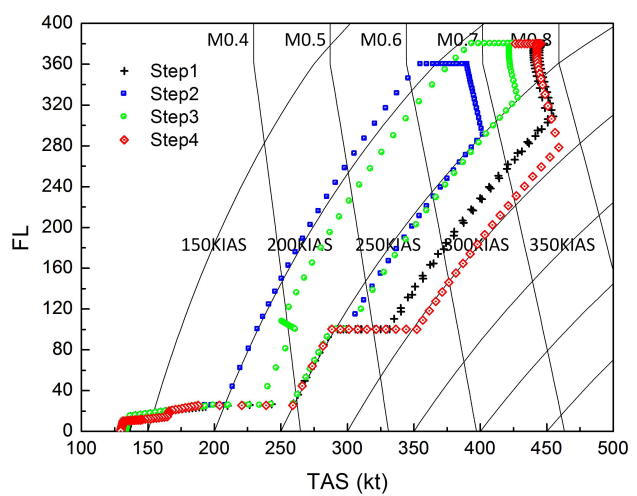

(b) Climb/descent speed profiles

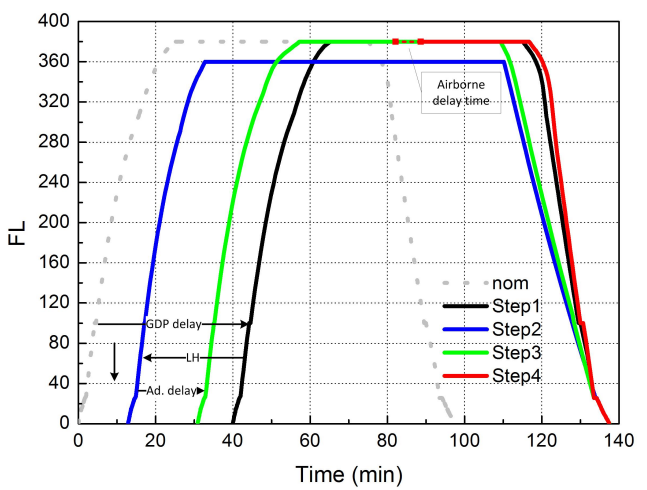

(d) Vertical trajectory vs. flight time

Figure 5: Resulting aircraft trajectories for each step of the study. 
Table 1: Summarized key parameters with respect to different flight phases.

\begin{tabular}{|c|c|c|c|c|c|c|c|c|c|c|c|c|c|c|c|c|c|}
\hline \multirow[b]{2}{*}{ Cases } & \multicolumn{2}{|l|}{ ATL } & \multicolumn{2}{|l|}{ Climb } & \multirow[b]{2}{*}{$\begin{array}{l}\text { Fuel } \\
(\mathrm{kg})\end{array}$} & \multicolumn{5}{|c|}{ Criuse } & \multicolumn{4}{|c|}{ Descent } & \multirow{2}{*}{$\begin{array}{c}\text { LGA } \\
\text { Slot } \\
\text { (hh:mm:ss) }\end{array}$} & \multicolumn{2}{|c|}{ Total } \\
\hline & $\begin{array}{c}\text { Slot } \\
\text { (hh:mm:ss) }\end{array}$ & $\begin{array}{l}\text { Dist } \\
(\mathrm{nm})\end{array}$ & $\begin{array}{l}\text { Speed } \\
(\mathrm{k} / \mathrm{kt} / \mathrm{M})\end{array}$ & $\begin{array}{l}\text { Time } \\
(\mathrm{min})\end{array}$ & & $\begin{array}{c}\text { AD Slot } \\
\text { (hh:mm:ss) }\end{array}$ & $\begin{array}{l}\text { Dist } \\
(\mathrm{nm})\end{array}$ & $\begin{array}{l}\text { Speed } \\
\text { (M) }\end{array}$ & $\begin{array}{l}\text { Time } \\
\text { (min) }\end{array}$ & $\begin{array}{l}\text { Fuel } \\
(\mathrm{kg})\end{array}$ & $\begin{array}{l}\text { Dist } \\
(\mathrm{nm})\end{array}$ & $\begin{array}{l}\text { Speed } \\
(\mathrm{M} / \mathrm{kt} / \mathrm{kt})\end{array}$ & $\begin{array}{l}\text { Time } \\
\text { (min) }\end{array}$ & $\begin{array}{c}\text { Fuel } \\
(\mathrm{kg})\end{array}$ & & $\begin{array}{l}\text { Fuel } \\
(\mathrm{kg})\end{array}$ & $\begin{array}{l}\text { Time } \\
(\mathrm{min})\end{array}$ \\
\hline nom & 00:00:00 & 162.2 & 250/288/0.77 & 25.2 & 1786 & $00: 47: 56$ & 372.4 & 0.78 & 49.9 & 1831 & 127.7 & $0.77 / 286 / 250$ & 22.4 & 327 & $01: 37: 28$ & 3945 & 97.5 \\
\hline Step1 & $00: 40: 00$ & 162.2 & 250/288/0.77 & 25.2 & 1786 & $01: 27: 56$ & 372.4 & 0.78 & 49.9 & 1831 & 127.7 & $0.77 / 286 / 250$ & 22.4 & 327 & 02:17:28 & 3945 & 97.5 \\
\hline Step2 & $00: 12: 53$ & 112.7 & 250/259/0.68 & 19.9 & 1467 & $00: 59: 24$ & 434.2 & 0.67 & 77.3 & 2205 & 115.4 & $0.62 / 201 / 201$ & 27.3 & 273 & 02:17:28 & 3945 & 124.6 \\
\hline Step3 & $00: 30: 53$ & 161.2 & $250 / 261 / 0.74$ & 26.4 & 1751 & $01: 21: 26$ & 368.6 & 0.74 & 52.0 & 1779 & 132.4 & $0.69 / 214 / 225$ & 28.2 & 310 & 02:17:28 & 3840 & 106.6 \\
\hline Step4 & $00: 30: 53$ & 161.2 & 250/261/0.74 & 26.4 & 1751 & $01: 28: 26$ & 379.6 & 0.78 & 52.4 & 1867 & 121.4 & $0.77 / 306 / 250$ & 20.8 & 320 & 02:17:28 & 3938 & 99.6 \\
\hline
\end{tabular}

Fig. 5(a) plots the vertical trajectory and true airspeed (TAS) versus flight distance for each Step. It can be observed that in order to realize the maximum LH, the best solution is to fly at a lower cruise flight level (FL) than the nominal flight (from FL380 to FL360). In general, as the cruise speed reduces to perform LH (see the TAS of Step2), the optimal flight level decreases, to achieve a higher specific range (lower fuel consumption). However, when pre-departure additional delays are experienced, the required LH is neutralized from the maximum, leading less speed reduction (see the TAS of Step3), and due to the discrete FL allocation scheme (increments of 2000ft), the actual trajectory remains at its initial altitude (FL380).

With regards to climb and descent phases, the lower the speed is, the steeper the climb and the flatter the descent will be, as can be noticed in Fig. 5(a). Climb and descent speeds, however, are not continuous in TAS (see Sec. 3). Instead, they are performed mainly from a continuous ac/deceleration process at low altitudes, a constant CAS climb/descent, followed by a constant Mach climb/descent over the crossover altitude, as shown in Fig. 5(b), with the opposite order for climb and descent (see Speed in Table. 1).

Through an airborne delay, the required LH continues to decrease, with a speed increase observed (see the TAS for Step4) compared to the Step3 for the remaining trajectory, which is even higher than the nominal, as seen also in Fig. 5(b), 305kt than 286kt in constant CAS descent. Nevertheless, recall that in Sec. 2 we emphasize that within the margin between the nominal speed and equivalent speed, no extra fuel is allowed (see Fig. 2), and in Step4, the descent speed seems out of this margin, while not burning extra fuel, as shown with $3938 \mathrm{~kg}$ in Table. 1. This is because before the airborne delay, some fuel has been saved in Step3, and if nothing happens the total fuel consumption will be lower than initially scheduled (see Fig. 5(c)), such that it is feasible to have this part of saved fuel consumed for the rest of the flight to maintain a higher speed. 
Fig. 5(d) illustrates the changes on flight timeline for the different optimization steps, where we can first observe a parallel shift from nominal flight to Step1 with a length of GDP delay (40mins). It can be also observed that Step2 departs 27mins earlier than Step1 (maximum LH) whilst keeping the same arrival time (i.e., the total flight time is extended by $27 \mathrm{mins}$ ). However, the total flight time shrinks in Step3 due to the additional pre-departure delays (18mins). Finally, after an airborne delay lasting 7mins (see Step4 in Table. 1), the arrival time still remains the same. That is to say, the additional delays (25mins in total) in this case study are entirely recovered at final arrival, and at no extra fuel cost.

In order to see how much delay recovery can be realized when having different combinations of pre- and post-departure delays, more computational experiments have been performed in the same scenario, changing the value for each delay. Results are as shown in Fig. 6.

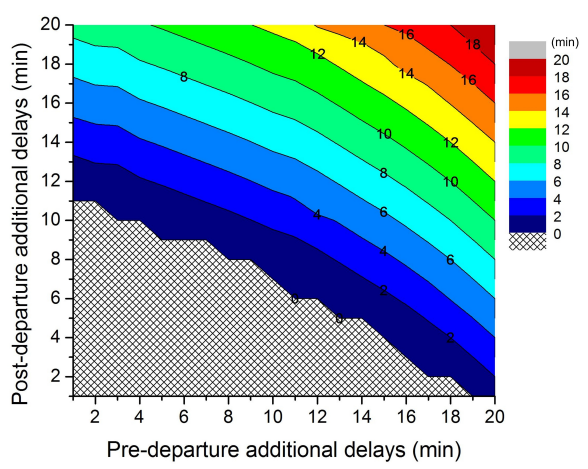

(a) GDP delay of 20 minutes

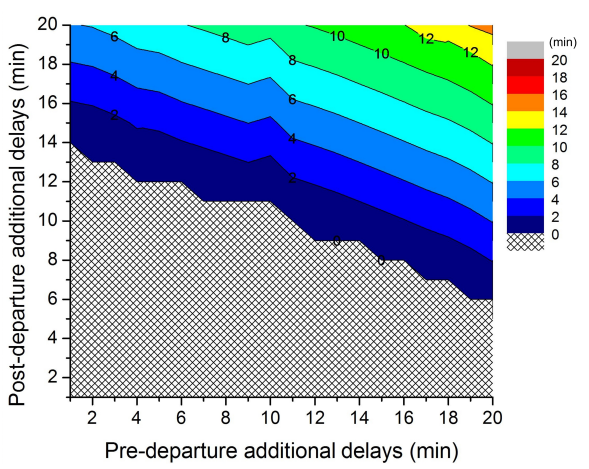

(b) GDP delay of 40 minutes

Figure 6: Extent of delay recovery (shown with different color) in response to combinations of pre- and post-departure delays at no extra fuel cost.

Two lengths of GDP delay are considered, 20mins and 40mins, where the former is lower than the maximum LH (27mins) of this particular flight, while the latter higher. Fig. 6 shows the actual additional delays experienced at the arrival as a function of pre- and post-departure delays, both of which range from 0 to $20 \mathrm{mins}$ with a step of $1 \mathrm{~min}$. Each color strip represents an interval in 2mins, and the shaded area highlights those combinations where no additional delay is realized.

For the GDP delay of 20mins, since the updated departure time cannot be prior to initially scheduled (see Eq. 7), which restrains the effect of an 
earlier departure time (Step2) enabled by LH to neutralize additional delays, we can see the delay recovery is limited to some extent compared with that in GDP delay of 40mins (compare Fig. 6(a) and 6(b)).

In both of the cases, it seems that more delay recovery can be yielded with respect to pre-departure than post-departure delays, because there is obviously more space and time for LH (to adjust speed) during the whole flight, rather than partially after the unforeseen delays en route. For the same reason, the contour lines turn to be flatter in areas where high postdeparture and low pre-departure delays occur, if compared to the opposite areas within the same stripe of additional delays.

Finally, it is worth noting some bumps on the contour lines, where the pre-departure delay equals to 3mins and 10mins in Figs. 6(a) and 6(b), respectively. Recall again the trade-offs between fuel consumption and flight time shown in Fig. 2. Any LH lower than the maximum contributes to saving some fuel. Therefore, when a specific LH is performed at the same time having the minimum fuel consumed, the saved fuel can be burned at the most to increase flight speed after airborne delays, in such a way to trade for a relatively higher delay recovery.

\section{Conclusions}

This paper focused on a problem recently drawing a growing body of research in air transportation, which was about the additional delays experienced by flights subject to ground holding. Inspired from previous works on linear holding (LH) for airborne delay absorption, its potential applicability was proposed to neutralize the additional delays at no extra fuel consumption. Illustrative examples were given in a scenario of the NAS in the United States, particularly with the GDPs (or AFPs) which are one of the most sophisticated ATFM protocols currently in use. But the usability of the proposed strategy should not be limited in the examples as such, given that the essentials of additional delays (i.e., uncertainties) might apply for any ATFM scenario and the LH could always help to enhance the traffic flow predictability and thus, lower the uncertainties.

Through multiple stages of optimal trajectory generation, LH was enabled to be implemented along the whole flight phases, and adjusted flexibly in response to different kinds of TMIs and the amounts of unforeseen delays they produce. Compared to the case where ground holding is fully endured followed by burning more fuel to increase flight speed to partially recover 
delays (as usually done nowadays for some airlines), the proposed LH strategy in this paper can reduce the additional delays without consuming any extra fuel than initially scheduled.

While the LH strategy proved to be efficient in delay recovery, as results suggest, one premise must be noted, which is time-of-arrival control in the trajectory in order to enforce the full assigned delay at the destination, as conveyed in the concept of trajectory based operations. Otherwise, airlines may be prone to depart intentionally earlier in order to compete for the reduced (and not enforced) available arrival slots, somehow aggravating traffic congestions, as has been identified in [2], as one of the main contributors to double delays.

Future work will aim at the simulation in realistic scenarios. Since the GDP/AFP is typically issued under severe weather conditions, the wind and non-standard atmospheres (which always have a great effect on real flights) should be taken into consideration too. In addition, after suffering long delays, the operators may be inclined to burn extra fuel than initially scheduled to expect more delay recovered. Thus, further defining a relation between the amount of extra fuel and the extent of delay recovery would be helpful for airlines in decision making.

\section{Acknowledgment}

The authors would like to thank Airbus Industrie for the use of PEP (Performance Engineers Program) suite, which allowed us to undertake realistic aircraft performances simulations. This research is partially supported by grants from the Funds of China Scholarship Council (201506830050). The

authors would like to thank the reviewers and the editor for their comments that have improved the paper.

\section{References}

[1] K. Bilimoria, "Analysis of additional delays experienced by flights subject to ground holding," in Proceedings of the 16th AIAA Aviation Technology, Integration, and Operations Conference, (Washington, D.C., USA), 2016.

[2] A. D. Evans and P. U. Lee, "Analyzing double delays at newark liberty international airport," in Proceedings of the 16th AIAA Aviation Tech- 
nology, Integration, and Operations Conference, (Washington, D.C., USA), 2016.

[3] M. Dwyer, L. Epstein, A. Futer, M. Hogan, K. Howard, R. Oisen, and B. Sharick, Interactions of Multiple Traffic Management Initiatives: An Initial Analysis, Version 2, 2011.

[4] S. Grabbe, B. Sridhar, A. Mukherjee, and A. Morando, "Traffic management advisor flow programs: an atlanta case study," in Proceedings of the AIAA Guidance, Navigation, and Control Conference, (Portland, USA), pp. 8-11, 2011.

[5] J. Rebollo and C. Brinton, "Brownian motion delay model for the integration of multiple traffic management initiatives," in Proceedings of the 11th USA/Europe Air Traffic Management Research and Development Seminar (ATM2015), (Lisbon, Portugal), 2015.

[6] L. Delgado, X. Prats, and B. Sridhar, "Cruise speed reduction for ground delay programs: A case study for san francisco international airport arrivals," Transportation Research Part C: Emerging Technologies, vol. 36, pp. 83-96, 2013.

[7] L. Delgado and X. Prats, "Effect of wind on operating-cost-based cruise speed reduction for delay absorption," Intelligent Transportation Systems, IEEE Transactions on, vol. 14, no. 2, pp. 918-927, 2013.

[8] L. Delgado and X. Prats, "Operating cost based cruise speed reduction for ground delay programs: Effect of scope length," Transportation Research Part C: Emerging Technologies, vol. 48, pp. 437-452, 2014.

[9] J. C. Jones, D. J. Lovell, and M. O. Ball, "En route speed control methods for transferring terminal delay," in Proceedings of the 10th USA/Europe Air Traffic Management Research and Development Seminar, (Chicago, USA), 2013.

[10] J. C. Jones, D. J. Lovell, and M. O. Ball, "Combining control by cta and dynamic en route speed adjustment to improve ground delay program performance," in Proceedings of the 11th USA/Europe Air Traffic Management Research and Development Seminar, (Lisbon, Portugal), 2015. 
[11] T. Günther and H. Fricke, "Potential of speed control on flight efficiency," in Proceedings of the 2nd International Conference on Research in Air Transportation (ICRAT), vol. 1, (Belgrade, Serbia), pp. 197-201, 2006.

[12] C. Tomlin, G. J. Pappas, and S. Sastry, "Conflict resolution for air traffic management: A study in multiagent hybrid systems," IEEE Transactions on automatic control, vol. 43, no. 4, pp. 509-521, 1998.

[13] Y. Xu, R. Dalmau, and X. Prats, "Effects of speed reduction in climb, cruise and descent phases to generate linear holding at no extra fuel cost," in Proceedings of the "th International Conference on Research in Air Transportation (ICRAT), (Philadelphia, USA), 2016.

[14] FAA, Traffic Flow Management in the National Airspace System, FAA2009-AJN-251, 2009.

[15] H. Balakrishnan and Y. Jung, "A framework for coordinated surface operations planning at dallas-fort worth international airport," in Proceedings of the AIAA Guidance, Navigation and Control Conference, (Hilton Head, USA), 2007.

[16] H. Khadilkar and H. Balakrishnan, "Network congestion control of airport surface operations," Journal of Guidance, Control, and Dynamics, vol. 37, no. 3, pp. 933-940, 2014.

[17] Airbus, Getting to grips with the cost index, Issue II. Flight Operations Support and Line Assistance (STL), Customer Services Directorate, Blagnac, 1998.

[18] R. Dalmau and X. Prats, "Fuel and time savings by flying continuous cruise climbs: Estimating the benefit pools for maximum range operations," Transportation Research Part D: Transport and Environment, vol. 35, pp. 62-71, 2015.

[19] Airbus, Flight Crew Operation Manual (FCOM): A320, Version 1.3.1, 1993.

[20] J. T. Betts, Practical methods for optimal control and estimation using nonlinear programming, vol. 19. SIAM, 2010. 
[21] G. D. Corporation, "General Algebraic Modeling System (GAMS) Release 24.2.1." Washington, DC, USA, 2013.

[22] GAMS Development Corporation, Washington, DC, USA, GAMS - The Solver Manuals, GAMS Release 24.2.1, 2013.

[23] FAA, Pilot's Handbook of Aeronautical Knowledge, FAA-H-8083-25A, Chapter Glossary, Holding Pattern, 2016. 